\title{
WEAKLY COMPACT OPERATORS AND THE STRICT TOPOLOGIES
}

\author{
JosÉ AgUayo AND José SÁNCHEZ
}

\begin{abstract}
Let $X$ be a completely regular space. We denote by $C_{b}(X)$ the Banach space of all real-valued bounded continuous functions on $X$ endowed with the supremum-norm.

In this paper we prove some characterisations of weakly compact operators defined from $C_{b}(X)$ into a Banach space $E$ which are continuous with respect to $\beta_{t}, \beta_{\tau}$ and $\beta_{\sigma}$ introduced by Sentilles.
\end{abstract}

We also prove that $\left(C_{b}(X), \beta_{i}\right), i=t, \tau, \sigma$, has the Dunford-Pettis property.

\section{INTRODUCTION AND NOTATIONS}

In this paper, $E$ denotes a Banach space, $X$ a completely regular Hausdorff space, $C_{b}(X)$ the set of all continuous bounded real-valued functions on $X, \mathcal{F}$ the algebra generated by zero sets, that is, sets of the form $f^{-1}(0), f \in C_{b}(X)$, and $B a(X)$ the $\sigma$-algebra generated by zero-sets.

On $C_{b}(X)$ there are three important topologies, the so-called strict topologies, which are denoted by $\beta_{t}, \beta_{\tau}, \beta_{\sigma}$; the dual of $\left(C_{b}(X), \beta_{i}\right)$ is $M_{i}(X)$, for $i=t, \tau, \sigma$ (these topologies and duals are discussed in [5]). It is known that $\beta_{t} \leqslant \beta_{\tau} \leqslant \beta_{\sigma} \leqslant\|\|_{\infty}$ and they have the same bounded sets [5].

Let $\mathcal{A}$ be an algebra of subsets of $X$ and let $m: \mathcal{A} \rightarrow E$ a finite additive vectormeasure. We shall say that $m$ is strongly additive if the series $\operatorname{\Sigma } m\left(A_{n}\right)$ converges for every disjoint sequence $\left(A_{n}\right)_{n \in N}$ of elements of $\mathcal{A}$.

The set-functions of $\mathcal{A}$ into $\mathrm{R}$ defined by $v(m)(A)=\sup \left\{\Sigma\left\|m\left(A_{i}\right)\right\| A_{i} \in \mathcal{A}, A_{i} \cap\right.$ $\left.A_{j}=\emptyset, i \neq j, A=\cup A_{i}, i=1 \ldots n, n \in N\right\}$ and by

$$
\begin{aligned}
& \|m\|(A)=\sup \left\{\left\|\Sigma \alpha_{i} m\left(A_{i}\right)\right\| A_{i} \in \mathcal{A}, A_{i} \cap A_{j}=\emptyset, i \neq j, A=\cup A_{i}, i=1 \ldots n,\right. \\
& \left.n \in N,\left|\alpha_{i}\right| \leqslant 1\right\}
\end{aligned}
$$

are called the variation and semi-variation of $m$, respectively. If $v(m)(X)(\|m\|(X))$ is finite, we shall say that $m$ is of bounded variation (semi-variation). It is known ([1]) that $\|m\|(A)=\sup \left\{\left|x^{\prime} \circ m\right|(A) \mid x^{\prime} \in E^{\prime},\left\|x^{\prime}\right\| \leqslant 1\right\}$. We will denote by $b a(\mathcal{A}, E)$ the space of all bounded semi-variation vector measures $m$ of $\mathcal{A}$ into $E$. $b a(\mathcal{A}, E)$ is a Banach space with the norm $m \rightarrow\|m\|(X)$.

If $S(\mathcal{A})$ denotes the space of all simple functions with the supremum-norm, then for each $m \in b a(\mathcal{A}, E)$, we define $T_{m}(f)=\Sigma \alpha_{i} m\left(A_{i}\right)$, where $f=\Sigma \alpha_{i} \chi_{A_{i}} \in S(\mathcal{A})$. Hence

Received 15 July 1988

Copyright Clearance Centre, Inc. Serial-fee code: 0004-9729/89 \$A2.00+0.00. 
$T_{m}$ is a continuous linear operator of $S(\mathcal{A})$ into $E$ and $\|T\|=\|m\|(X)$. Conversely, each continuous linear operator $T$ of $S(\mathcal{A})$ into $E$ defines a bounded vector measure of semi-variation $m$, with $m(A)=T\left(\chi_{A}\right)$.

Let $B(\mathcal{A})$ denote the completion of $S(\mathcal{A})$ in the supremum-norm. It is proved in [1] that the mapping $m \rightarrow T_{m}$ is an isomorphism of the space $b a(\mathcal{A}, E)$ with the space $L(B(\mathcal{A}), E) . T_{m}(f)$ is denoted by $\int f d m$.

We shall say that an operator $T$ in $L(B(\mathcal{A}), E)$ is weakly compact if $T$ maps any bounded subset of $B(\mathcal{A})$ to a relatively weakly compact subset of $E$. The following theorem is proved in [1].

ThEorem 1.1. Let $T: B(\mathcal{A}) \rightarrow E$ be a bounded linear operator. Then the following are equivalent:

(i) $T$ is weakly compact

(ii) $m$ is strongly additive

The space of all finite, finitely additive, zero set regular, real-valued measures is denoted by $M(X)$ (zero set regular means that for any $\epsilon>0$ and any $F \in \mathcal{F}$, there exist a zero set $Z$ and a cozero set $U$, with $Z \subseteq F \subseteq U$, such that $|\mu|(U \backslash Z)<\epsilon)$.

Alexandroff's Theorem $([\boldsymbol{\theta}])$ establishes that $M(X)$ is the dual of $C_{b}(X)$ with the supremum-norm.

As in the case when $X$ is a compact Hausdorff space we can identify $B(\mathcal{F})$ with $M(X)^{\prime}$ by the isometry $f \rightarrow \lambda_{f}$ where $\lambda_{f}(\mu)=\int_{X} f d \mu$.

LEMMA 1.2. Each $f \in C_{b}(X)$ is the uniform limit of simple functions, that is $C_{b}(X) \subseteq B(\mathcal{A})$.

Proof: First note that if $f \in C_{b}(X),\{x \in X: f(x) \geqslant \alpha\}$ is a zero set. Let $\epsilon>0$; since $f(X)$ is totally bounded, there is a finite subset $\left\{x_{1}, x_{2}, \ldots x_{n}\right\}$ of $X$ such that $f(X) \subseteq \cup f\left(x_{i}\right)+(-\epsilon, \epsilon)$.

Write $U_{i}=f\left(x_{i}\right)+(-\epsilon, \epsilon)$ and $A_{i}=f^{-1}\left(U_{i}\right)$. If the family $\left\{A_{i}: i=1, \ldots, n\right\}$ is not disjoint, then we define $B_{1}=A_{1}$ and $B_{i}=A_{i} \backslash B_{i}, i=2, \ldots, n$; thus, $\left\{B_{i} \mid\right.$ $i=1, \ldots n\}$ is a $\mathcal{F}$-partition of $X$. If $f_{0}=\Sigma f\left(x_{i}\right) \chi_{B_{i}}$ and if $x \in X$, then there exists $i \in\{1, \ldots, n\}$ such that $x \in A_{i} \backslash B_{i}$ and $f(x)=f\left(x_{i}\right)+u$, with $|u|<\epsilon$, which implies that $\left|f(x)-f\left(x_{i}\right)\right|<\epsilon$. Thus, $\left|f(x)-f_{0}(x)\right|<\epsilon$ and then $\left\|f-f_{0}\right\|<\epsilon$ since $x$ was arbitrary.

TheOREM 1.3. Let $T: C_{b}(X) \rightarrow E$ be a bounded linear operator. Then, there exists a unique finitely additive vector measure $m: \mathcal{F} \rightarrow E^{\prime \prime}$ of bounded semi-variation such that:

(a) for any $x^{\prime} \in E^{\prime}, x^{\prime} \circ m \in M(X)$; 
(b) the mapping of $E$ into $M(X)$ defined by $x^{\prime} \rightarrow x^{\prime} \circ m$ is $\sigma\left(E^{\prime}, E\right)-$ $\sigma\left(M(X), C_{b}(X)\right)$ continuous;

(c) $T(f)=\int_{X} f d m, \forall f \in C_{b}(X)$;

(d) $\|T\|=\|m\|(X)$.

Conversely, if $m: \mathcal{F} \rightarrow E^{\prime \prime}$ is a finitely additive vector measure of bounded semivariation satisfying (a) and (b), then (c) defines a bounded linear operator $T: C_{b}(X) \rightarrow$ $E$ such that $\|T\|=\|m\|(X)$.

Proof: Since $T^{\prime \prime}: C_{b}(X)^{\prime \prime} \rightarrow E^{\prime \prime}$ is a bounded linear operator and $B(\mathcal{F}) \subset$ $C_{b}(X)^{\prime \prime}$, there is a vector measure associated with $\bar{T}=T_{\mid B(F)}^{\prime \prime}$. Since $C_{b}(X) \subseteq B(\mathcal{F})$, we have that, for each $f \in C_{b}(X)$,

$$
T(f)=\bar{T}(f)=\int_{X} f d m \text { and }\|T\| \leqslant\|\bar{T}\|=\|m\|(X) \leqslant\left\|T^{\prime \prime}\right\|=\|T\| .
$$

Part (a) and (b) follow easily from the continuity of $T$ and $T^{\prime}$.

Conversely, since $\left\{x^{\prime} \circ m:\left\|x^{\prime}\right\| \leqslant 1\right\}$ is $\sigma\left(M(X), C_{b}(X)\right)$ relatively compact, we have that

$$
\|m\|(X)=\sup \left\{\left|x^{\prime} \circ m\right|(X):\left\|x^{\prime}\right\| \leqslant 1\right\}<\infty
$$

and then $m$ is of bounded semi-variation. The result follows from this.

\section{WEAKLY COMPACT OPERATORS AND STRICT TOPOLOGIES}

In this section we shall study the weakly compact operators of $C_{b}(X)$ into $E$ which are continuous in the strict topologies $\beta_{t}, \beta_{\tau}$ and $\beta_{\sigma}$, respectively, and their associated vector measures.

We already know that if $T$ is weakly compact, then $T^{\prime}$ from $E^{\prime}$ into $M(X)$ is also weakly compact; thus, $T^{\prime}\left(B_{E^{\prime}}\right)=\left\{x^{\prime} \circ m:\left\|x^{\prime}\right\| \leqslant 1\right\}$ is relatively $\sigma\left(M(X), C_{b}(X)^{\prime \prime}\right)$ compact and then $\left\{\left|x^{\prime} \circ m\right|:\left\|x^{\prime}\right\| \leqslant 1\right\}$ is relatively $\sigma\left(M(X), C_{b}(X)^{\prime \prime}\right)$-compact ([1]) which implies that $\left\{\left|x^{\prime} \circ m\right|:\left\|x^{\prime}\right\| \leqslant 1\right\}$ is relatively $\sigma\left(M(X), C_{b}(X)\right)$-compact.

The following theorems characterise the $\beta_{i}$-continuous, weakly compact linear operators, where $i=t, \tau, \sigma$.

From now on, we will assume that $T: C_{b}(X) \rightarrow E$ is a weakly compact operator.

THEOREM 2.1. If $m$ is the associated vector measure of $T$, then the following are equivalent:

(i) $m$ is $\sigma$-additive vector measure;

(ii) if $\left\{f_{n}\right\}_{n \in N}$ is any decreasing sequence in $C_{b}(X)$, with

$$
f_{n}(x) \rightarrow 0 \text { for each } x \in X, \text { then }\left\|T f_{n}\right\| \rightarrow 0 ;
$$

(iii) $T$ is $\beta_{\sigma}$-continuous. 
Proof: (i) $\Rightarrow$ (ii) Let $\left\{f_{n}\right\}_{n \in N}$ be a sequence in $C_{b}(X)$ such that $f_{n} \downarrow 0$ pointwise and $\epsilon>0$. By the Caratheodory-Hahn Extension Theorem ([1]), there exists a nonnegative real-valued $\sigma$-additive measure $\mu$ on $B a(X)$ such that $m<<\mu$. Thus, for the given $\epsilon$, there exists $\delta>0$ such that $\mu(A)<\delta$ implies $\left|x^{\prime} \circ m\right|(A)<\epsilon$ uniformly in $x^{\prime} \in B_{E^{\prime}}$.

On the other hand, Egoroff's Theorem gives us a subset $F \in B a(X)$ such that $f_{n} \rightarrow 0$ uniformly on $X \backslash F$ and $\mu(F)<\delta$. Therefore, there exists $n_{0} \in N$ such that $n \geqslant n_{0}$ implies $\left\|f_{n}\right\|<\epsilon / 2 M$ on $X \backslash F$, where $M=\|m\|(X)$.

Now, if $n \geqslant n_{0}$, then

$$
\begin{aligned}
\left|x^{\prime} \circ T\left(f_{n}\right)\right| & \leqslant \int_{X}\left|f_{n}\right| d\left|x^{\prime} \circ m\right| \\
& =\int_{X \backslash F}\left|f_{n}\right| d\left|x^{\prime} \circ m\right|+\int_{F}\left|f_{n}\right| d\left|x^{\prime} \circ m\right| \\
& <(\epsilon / 2 m)\left|x^{\prime} \circ m\right|(X \backslash F)+\left|x^{\prime} \circ m\right|(F) \\
& <\epsilon / 2+\epsilon / 2=\epsilon
\end{aligned}
$$

uniformly in $x^{\prime} \in \boldsymbol{B}_{E^{\prime}}$.

The conclusion follows from the fact that

$$
\|T f\|=\sup \left\{\left|x^{\prime} \circ T(f)\right|:\left\|x^{\prime}\right\| \leqslant 1\right\} .
$$

(ii) $\Rightarrow$ (iii) If $\left\|T f_{n}\right\| \rightarrow 0$ for any sequence $\left\{f_{n}\right\}_{n \in N}$ in $C_{b}(X)$, then $\left|x^{\prime} \circ m\right| \epsilon$ $M_{\sigma}(X)$ for all $x^{\prime} \in B_{E^{\prime}}$. Since $\left\{\left|x^{\prime} \circ m\right|:\left\|x^{\prime}\right\| \leqslant 1\right\}$ is relatively $\sigma\left(M(X), C_{b}(X)\right)$ compact, we have $\left\{\left|x^{\prime} \circ m\right|:\left\|x^{\prime}\right\| \leqslant 1\right\}$ is. relativley compact and then $\beta_{\sigma}$-equicontinuous [5, Theorem 5.2, p.322].

Let $\left\{f_{\alpha}\right\}_{\alpha \in I}$ be a net in $C_{b}(X)$ such that $f_{\alpha} \rightarrow 0$ with respect to $\beta_{\sigma}$. Hence $\left|x^{\prime} \circ T\right|\left(f_{\alpha}\right) \rightarrow 0$ uniformly in $x^{\prime} \in B_{E^{\prime}}$; since $\left|x^{\prime} \circ T\left(f_{\alpha}\right)\right| \leqslant\left|x^{\prime} \circ T\right|\left(f_{\alpha}\right)$, we get $\left|x^{\prime} \circ T\left(f_{\alpha}\right)\right| \rightarrow 0$ uniformly in $x^{\prime} \in B_{E^{\prime}}$ and then $\left\|T f_{\alpha}\right\|=\sup \left\{\left|x^{\prime} \circ T\left(f_{\alpha}\right)\right|\right.$ : $\left.\left\|x^{\prime}\right\| \leqslant 1\right\} \rightarrow 0$. Consequently, $T$ is continuous with respect to $\beta_{\sigma}$.

(iii) $\Rightarrow$ (i) Since $\left|x^{\prime} \circ T(f)\right| \leqslant\|T f\|\left\|x^{\prime}\right\|$ for all $x^{\prime} \in B_{E^{\prime}}$ and $T$ is continuous with respect to $\beta_{\sigma}$ we have that $x^{\prime} \circ T$ is continuous with respect to $\beta_{\sigma}$ for any $x^{\prime} \in B_{E^{\prime}}$; hence $\left|x^{\prime} \circ m\right|$ is a real-valued $\sigma$-additive measure for any $x^{\prime} \in B_{E^{\prime}}$. The conclusion follows from [1, Theorem 2 p.27].

The next theorem characterises the weakly compact operators which are continuous with respect to $\beta_{t}$. By Sentilles [5], $\beta_{t}$ is the finest locally convex topology on $C_{b}(X)$ agreeing with the compact-open topology on the norm-bounded subsets of $C_{b}(X)$.

ThEOREM 2.2. If $m$ is the associated vector measure of $T$, then the following are equivalent:

(i) $(\forall \epsilon>0)(\exists K \subset X, K$ compact $)(\|m\|(X \backslash K)<\epsilon)$; 
(ii) $T$ is continuous with respect to $\beta_{t}$;

(iii) $T$ is continuous on the unit ball with respect to the compact-open topology.

Proof: (i) $\Rightarrow$ (ii) If $\epsilon>0$ is given, then there exists a compact subset $K$ of $X$ such that $\left|x^{\prime} \circ m\right|(X \backslash K)<\epsilon$ uniformly in $x^{\prime} \in B_{E^{\prime}}$. Therefore $\left\{\left|x^{\prime} \circ m\right|:\left\|x^{\prime}\right\| \leqslant 1\right\}$ and then $\left\{x^{\prime} \circ m:\left\|x^{\prime}\right\| \leqslant 1\right\}$ is $\beta_{t}$-equicontinuous ([5]). Thus, $T$ is continuous with respect to $\beta_{t}$.

(ii) $\Rightarrow$ (iii) Follows from the definition of $\beta_{t}$.

(iii) $\Rightarrow$ (i) From the fact that $T$ is continuous with respect to $\beta_{t}$, we have that $\left\{\left|x^{\prime} \circ m\right|:\left\|x^{\prime}\right\| \leqslant 1\right\}$ is $\beta_{t}$-equicontinuous. The result follows from [5, Theorem 5.1].

THEOREM 2.3. If $m$ is the associated vector measure of $T$, then the following are equivalent:

(i) $T$ is continuous with respect to $\beta_{\tau}$;

(ii) for any decreasing net $\left\{f_{\alpha}\right\}_{a \in I}$ in $C_{b}(X)$ with

$$
f_{\alpha}(x) \rightarrow 0 \text { for each } x \in X,\left\|T f_{\alpha}\right\| \rightarrow 0 \text {; }
$$

(iii) for any net of zero sets $Z_{\alpha}$ decreasing to the null set, $\|m\|\left(Z_{\alpha}\right) \rightarrow 0$.

Proof: (i) $\Rightarrow$ (ii) If $\left\{f_{\alpha}\right\}_{\alpha \in I}$ is a decreasing net in $C_{b}(X)$ such that $f(x)_{\alpha} \rightarrow 0$, for each $x \in X$, then $f_{\alpha} \rightarrow 0$ in the topology $\beta_{\sigma}([6])$. Thus, $\left\|T f_{\alpha}\right\| \rightarrow 0$.

(ii) $\Rightarrow$ (i) Since $\left|x^{\prime} \circ T(f)\right| \leqslant\|T f\|\left\|x^{\prime}\right\|$, for any $x^{\prime} \in E^{\prime}$, we have that $x^{\prime} \circ T$ is $\tau$-additive and then $\left\{\left|x^{\prime} \circ m\right|:\left\|x^{\prime}\right\| \leqslant 1\right\}$ is relatively $\sigma\left(M_{\tau}(X), C_{b}(X)\right)$-compact. Therefore, $\left\{\left|x^{\prime} \circ m\right|:\left\|x^{\prime}\right\| \leqslant 1\right\}$ is $\beta_{\tau}$-equicontinuous $([5])$. The statement follows easily from this.

(i) $\Rightarrow$ (iii) Let $\left\{Z_{\alpha}\right\}_{\alpha \in I}$ be a net of zero sets decreasing to the null set. Consider $D=\left\{f \in C_{b}(X): 0 \leqslant f(x) \leqslant 1 \&(\exists \alpha)\left(f \equiv 1\right.\right.$ in $\left.Z_{\alpha}\right)$. We index the elements of $D$ as follows: $D=\left\{f_{\lambda}\right\}_{\lambda \in A}$ so that $\lambda>\mu$ if and only if $f_{\lambda} \leqslant f_{\mu}$. Thus $\left\{f_{\lambda}\right\}_{\lambda \in A}$ is a net in $C_{b}(X)$; further $f_{\lambda} \downarrow 0$. Hence $\left\|T f_{\alpha}\right\| \rightarrow 0$.

Since $\left|x^{\prime} \circ T(f)\right| \leqslant\|T f\|\left\|x^{\prime}\right\|$, we have that $x^{\prime} \circ m \in M_{\tau}(X)$ and $\left\{\left|x^{\prime} \circ m\right|:\left\|x^{\prime}\right\| \leqslant\right.$ $1\}$ is $\beta_{\tau}$-equicontinuous. From this we get that $\left|x^{\prime} \circ T\right|\left(f_{\lambda}\right) \rightarrow 0$ uniformly in $x^{\prime} \in B_{E^{\prime}}$.

Thus, if $\epsilon>0$ is given, then there exists $\lambda_{0} \in A$ such that $\lambda>\lambda_{0}$ implies $\left|x^{\prime} \circ T\right|\left(f_{\lambda}\right)=\int f_{\lambda} d\left|x^{\prime} \circ m\right|<\epsilon$ uniformly in $x^{\prime} \in B_{E^{\prime}}$. For this $\lambda_{0}$ there exists a $\alpha_{0} \in I$ so that $f \equiv 1$ on $Z_{\alpha_{0}}$ and

$$
\left|x^{\prime} \circ m\right|\left(Z_{\alpha}\right)=\int \chi z_{\lambda_{0}} d\left|x^{\prime} \circ m\right| \leqslant \int f_{\lambda_{0}} d\left|x^{\prime} \circ m\right|<\epsilon
$$

uniformly in $x^{\prime} \in B_{E^{\prime}}$. Therefore, if $\alpha>\alpha_{0},\left|x^{\prime} \circ m\right|\left(Z_{\alpha}\right)<\left|x^{\prime} \circ m\right|\left(Z_{\alpha_{0}}\right)<\epsilon$ uniformly in $x^{\prime} \in B_{E^{\prime}}$. The statement follows from the fact that $\|m\|(A)=$ $\sup \left\{\left|x^{\prime} \circ m\right|(A):\left\|x^{\prime}\right\| \leqslant 1\right\}$. 
(iii) $\Rightarrow$ (ii) Let $\left\{f_{\lambda}\right\}_{\lambda \in A}$ be a decreasing net in $C_{b}(X)$ such that $\left\|f_{\alpha}\right\| \leqslant 1$ and $f_{\alpha}(x) \rightarrow 0$ for each $x \in X$, and $\epsilon>0$. Define $Z_{\alpha}=\left\{x \in X: f_{\alpha}(x) \geqslant \epsilon / 2 M \& M=\right.$ $\|m\|(X)\} . \quad\left\{Z_{\alpha}\right\}_{\alpha \in I}$ is a decreasing net of zero sets such that $Z_{\alpha} \downarrow \emptyset$. Then there exists $\alpha_{0} \in I$ so that $\alpha>\alpha_{0}$ implies $\|m\|\left(Z_{\alpha}\right)<\epsilon / 2$.

Take $x^{\prime} \in E^{\prime},\left\|x^{\prime}\right\| \leqslant 1$, and $\alpha>\alpha_{0}$. Then

$$
\begin{aligned}
\left|x^{\prime} \circ T\left(f_{\alpha}\right)\right| & \leqslant \int_{X} f_{\alpha} d\left|x^{\prime} \circ m\right|=\int_{Z_{\alpha}} f_{\alpha} d\left|x^{\prime} \circ m\right|+\int_{X \backslash Z_{\alpha}} f_{\alpha} d\left|x^{\prime} \circ m\right| \\
& \leqslant\left|x^{\prime} \circ m\right|\left(Z_{\alpha}\right)+(\epsilon / 2 M)\left|x^{\prime} \circ m\right|\left(X \backslash Z_{\alpha}\right)<\epsilon
\end{aligned}
$$

uniformly in $x^{\prime} \in B_{E^{\prime}}$. Thus $T f_{\alpha} \rightarrow 0$.

We shall say that a topological vector space $E$ has the strict Dunford-Pettis property if for any Banach space $F$ and every linear continuous weakly compact operator $T$ from $E$ into $F$ transforms weakly Cauchy sequences into convergent sequences [1].

The following theorem applies the previous result to prove that $\left(C_{b}(X), \beta_{i}\right), i=$ $t, \tau, \sigma$, possess the strict Dunford-Pettis property. This result was proved by Khurana [4].

Theorem 2.4. $\left(C_{b}(X), \beta_{i}\right), i=t, \tau, \sigma$, possess the strict Dunford-Pettis property.

Proof: Since $\beta_{t} \leqslant \beta_{\tau} \leqslant \beta_{\sigma}$, it is enough to show the statement for the case $\left(C_{b}(X), \beta_{\sigma}\right)$.

Let $T$ be a linear $\beta_{\sigma}$-continuous operator of $C_{b}(X)$ to $E$ which is weakly compact. Thus, its associated vector measure $m$ is $\sigma$-additive and it admits a control measure $\mu$. So if $\epsilon>0$ is given, there exists $\delta>0$ such that $\mu(F)<\delta$ implies $\|m\|(F)<\epsilon$.

Let $\left\{f_{n}\right\}_{n \in N}$ be a weakly Cauchy sequence in $C_{b}(X)$. Then $\left\{f_{n}(x)\right\}_{n \in N}$ is Cauchy in $\mathbf{R}$ for each $x \in X$. By Egoroff's Theorem, there exists $F_{\delta} \in B a(X)$ such that $\left\{f_{n}\right\}_{n \in N}$ is Cauchy on $X \backslash F_{\delta}$ and $\mu\left(F_{\delta}\right)<\delta$.

Let $n_{0} \in N$ such that for $n, m \geqslant n_{0}$ we have $\sup \left\{\left\|f_{n}(x)-f_{m}(x)\right\|: x \in X \backslash F_{\delta}\right\}<$ $\epsilon / 2 M$, where $M=\|m\|(X)$. Thus

$$
\begin{aligned}
\left\|T f_{n}-T f_{m}\right\| & \leqslant\left\|\int_{X \backslash F_{\delta}}\left(f_{n}-f_{m}\right) d m\right\|+\left\|\int_{F_{\delta}}\left(f_{n}-f_{m}\right) d m\right\| \\
& \leqslant \sup \left\{\left\|f_{n}(x)-f_{m}(x)\right\|: x \in X\right\}\|m\|(X)+L\|m\|\left(F_{\delta}\right)<\epsilon,
\end{aligned}
$$

where $\left\|f_{n}\right\| \leqslant L$ for all $n \in N$.

\section{REFERENCES}

[1] J. Diestel and J. Uhl, Vector Measures (Surveys Number 15, American Math. Soc., Providence, 1977). 
[2] A. Grothendieck, 'Sur les applications Lineares faiblement compactes d'espaces du type $C(K)$ ', Canad. J. Math. 5 (1953), 129-173.

[3] P. Halmos, Measure Theory (Van Nostrand, Reinhold Co., 1969).

[4] S.S. Khurana, 'Dunford-Pettis Property', J. Math. Anal. Appl. 65 (1968), 361-364.

[5] D. Sentilles, 'Bounded Continuous Functions on a completely regular space', Trans. Amer. Math. Soc. 168 (1972), 311-336.

[6] R. Wheeler, 'A Survey of Baire measures and strict topologies', Exposition. Math. 1 (1983), 97-190.

Departmento de Matematica

Facultad de Ciencias

Universidad de Concepcion

Casilla 2017

Concepcion, Chile 